\title{
The environment of dwarf spheroidal satellites; ram pressure, tides and external radiation fields
}

\author{
Lucio Mayer \\ Institute of Theoretical Physics, University of Zürich, Winterthurerstrasse 190, 8057 Zurich, \\ Switzerland \\ email: lucio@physik.unizh.ch
}

\begin{abstract}
We discuss the role of environmental mechanisms in the evolution of dwarf galaxy satellites using high-resolution N-Body+SPH simulations that include simultaneously tidal forces, ram pressure and heating from ionizing radiation fields. Tidally induced bar-buckling instabilities can transform a rotating disky dwarf into pressure supported spheroidals. Efficient gas removal requires instead a combination of tidal mass loss and ram pressure stripping in a diffuse gaseous corona around the primary system. The efficiency of ram pressure depends strongly on how extended the gas remains during the evolution. Bar driven inflows that tend to drive the gas to the bottom of the potential well can be opposed by the heating from external radiation fields. We show that even fairly massive dwarfs $\left(V_{\text {peak }}>30 \mathrm{~km} / \mathrm{s}\right)$ would be stripped of their gas over a few Gyr if they enter the Milky Way halo at $z>2$ thanks to the effect of the cosmic UV background. Gas mass loss can be much faster, occurring in less than $1 \mathrm{Gyr}$, if dwarf satellites have their first close approach to the primary at the epoch of bulge formation. Indeed at that time the primary galaxy should have a FUV luminosity comparable to that of major present-day starbursts, resulting in a local UV field even more intense than the cosmic background.
\end{abstract}

Keywords. galaxies:dwarf;galaxies: evolution; cosmology: dark matter

\section{Introduction}

Dwarf spheroidals (dSphs) are the faintest galaxies known. They are gas poor and have pressure supported stellar components (Mateo 1998). Among them some stopped forming stars about 10 Gyr ago and other have extended star formation histories (Hernandez et al. 2000). They are typically clustered around the largest galaxy in a group. Both mass loss from supernovae winds (Dekel \& Silk 1986) and environmental mechanisms like tidal and ram pressure stipping (Einasto et al. 1974) have been invoked to explain their properties. Suppression of gas accretion and/or photoevaporation during the reionization epoch likely played a role as well (Bullock et al. 2000). In this paper we describe the results of N-Body/SPH simulations of disky dwarf galaxies subject to the combined action of the main environmental mechanisms, tides, ram pressure, and both a cosmic and local ultraviolet radiation field. The model galaxies and the choice of the orbits are consistent with the predictions of LCDM models (Mayer et al. 2002, 2005, hereafter MA05). These simulations allow for the first time to explore the overall effect of the environment.

\section{The masses of dwarf spheroidals}

Knowing the present and past mass of dSphs is crucial in order to compute the effects of both environmental and internal mechanisms that might affect their evolution. Moreover, 


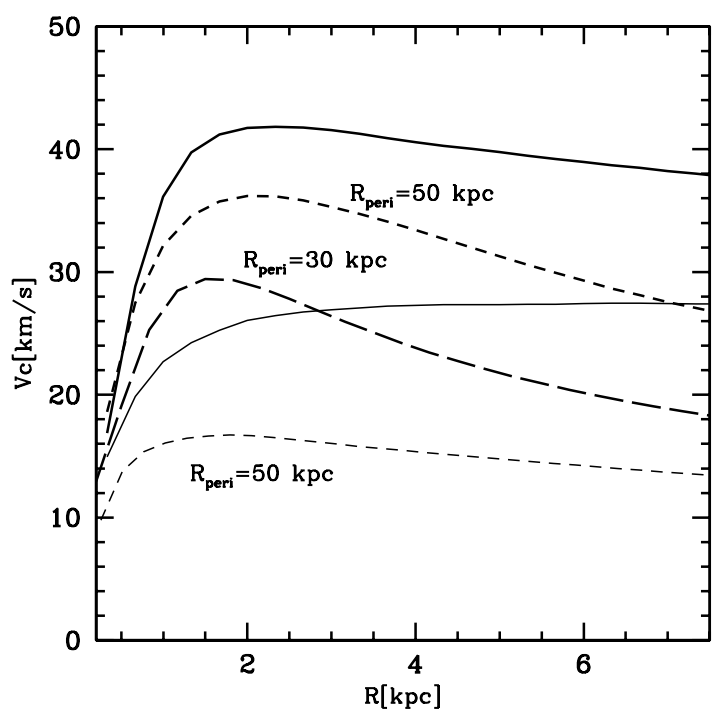

Figure 1. Evolution of the rotation curves of dwarf models with $V_{\text {peak }}=40 \mathrm{~km} / \mathrm{s}$ (thick lines)and $V_{\text {peak }}=28 \mathrm{~km} / \mathrm{s}$ (thin lines). The solid lines represent the initial conditions while the short-dashed show the curves after 3 orbits. The thick long-dashed line shows the curve for the same dwarf model with $V_{\text {peak }}=40 \mathrm{~km} / \mathrm{s}$ on a different orbit. Pericenter distances for the various orbits are indicated in the plot (see MA05)

the mass of dSphs has important implications on the missing satellites problem (Moore et al. 1999), namely the fact that CDM predicts an order of magnitude more satellites than observed around the Milky Way or M31. White (2001) has suggested that this problem could be alleviated if the true circular velocities of the halos of dSphs, and thus their masses, are higher than those obtained from their central velocity dispersion under the assumption of a flat (isothermal) rotation curve. This is expected if dSphs sit at the center of an extended halo with an non-isothermal (e.g. NFW) profile, thus having circular velocity that keep rising well beyond the apparent tidal radius of the galaxy. Stoehr et al. (2002) fitted the kinematics of dSphs inside subhaloes of a cosmological $\Lambda \mathrm{CDM}$ simulation using Jeans modeling and King profiles for the light distribution of dSphs and found that these galaxies likely live in halos having a peak circular velocity $V_{\text {peak }}=40-60 \mathrm{~km} / \mathrm{s}$. The halo density profiles employed in their analysis are much flatter than and NFW profile at the scale of the core radius of dSphs.

Kazantzidis et al. (2004) have studied the tidal disruption of individual CDM satellites with as many as $10^{7}$ particles and of subhaloes in a hi-res LCDM simulation. They repeated the analysis of Stoehr et al. (2002) and found that the observed velocity dispersions of Draco and Fornax can only be reproduced in subhaloes with $V_{\text {peak }}=20-30 \mathrm{~km} / \mathrm{s}$. In their simulations halos maintain cuspy profiles down to the force resolution, which is smaller than the core radius of dSphs $(\sim 100 \mathrm{pc})$. As a result the rotation curves rise more steeply than those of Stoehr et al. (2002), explaining the discrepancy. The masses of the tidally truncated subhaloes of Kazantzidis et al. (2004) are in the range $10^{8}-10^{9} M_{\odot}$, in agreement with more recent analysis based on newer data on stellar velocities (e.g. Wilkinson et al. (2001). These masses are still quite high, yielding $M / L>100$ for Draco. 


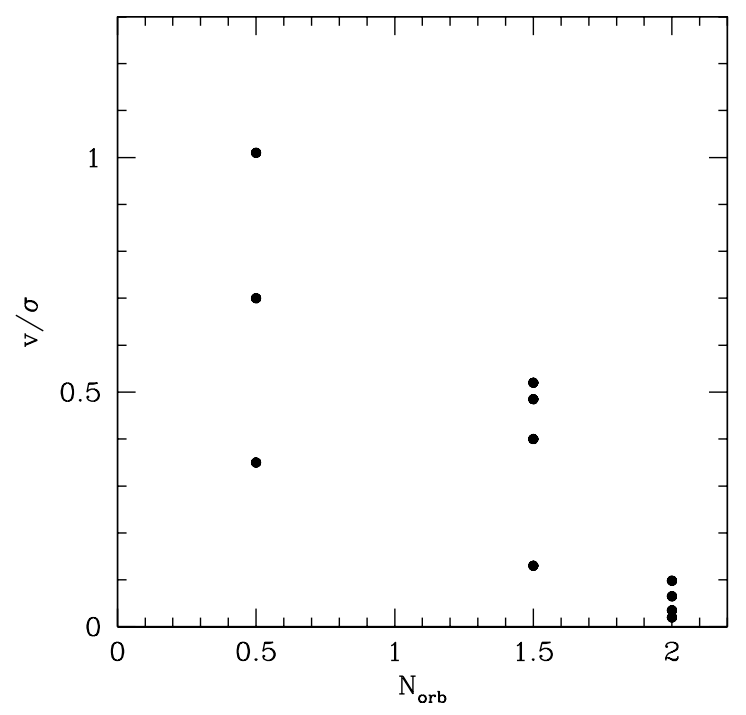

Figure 2. $v / \sigma$ as a function of the number of orbits for the satellites in the cosmological hydrodynamical simulation of Governato, Mayer et al. (2004). $v / \sigma$ is measured within the half mass radius of the dwarfs. The mean number of orbits for each of the three groups of dwarfs appears on the horizontal axis.

However peak circular velocities are at most a factor of 2 higher than those used in Moore et al. (1999), with the consequence that little changes for the missing satellite problem.

The initial $V_{\text {peak }}$ of dSphs was larger than $30 \mathrm{~km} / \mathrm{s}$ since mass loss produced by tidal shocks can lower $V_{\text {peak }}$ (Ghigna et al. 1998). Kravtsov et al. (2004)), using hi-res cosmological simulations without baryons, find that $V_{\text {peak }}$ can drop by a factor of 2 in 10 Gyr or so. Mayer et al. (2002) and MA05 always find a smaller decrease in $V_{\text {peak }}$ in their large set of SPH simulations that follow the evolution of satellites with baryonic disks embedded in CDM haloes (Figure 1). The different results might be due to the fact that some of the satellites in Kravtsov et al. (2004) have orbits with pericenters smaller than those considered by MA05. However, the inclusion of baryons also makes the satellites more robust to the tidal disruption of the inner region, which is what matters for the evolution of $V_{\text {peak }}$. Whether or not the baryonic disk becomes bar unstable also is important, since bar-driven inflows can deepen the central potential of the dwarf (Kazantzidis, Mayer et $a l$, in preparation). High-resolution cosmological simulations with hydrodynamics will eventually be able to quantify the overall significance of these effects. In any case, the results of MA05 suggest that the initial $V_{\text {peak }}$ of dSphs was in the range $35-50 \mathrm{~km} / \mathrm{s}$. This has important implications. First, it means that photoevaporation did not play a major role during the early evolution of satellites at high $z$ since it is only effective for $V_{\text {peak }}<20 \mathrm{~km} / \mathrm{s}$ (Susa \& Umemura 2004; Shaviv \& Dekel 2003). This is consistent with the lack of a clear signature of the reionization epoch in the star formation histories of dSphs (Grebel \& Gallagher 2004). Second, blow-out of most of the gas mass due to supernovae winds cannot have occurred since it requires $V_{\text {peak }}<30 \mathrm{~km} / \mathrm{s}$ (e.g. MacLow \& Ferrara 1999). 


\section{Morphological evolution of disky dwarfs into dSphs; tidal stirring}

Aside from the different gas content, three other facts have to be considered when comparing dSphs and gas-rich, similarly faint dwarf galaxies known as dwarf irregulars (dIrrs). First, except for the faintest among them all dirrs in the Local Group and nearby groups exhibit substantial rotation while dSphs do not. Second, stellar profiles in the two classes of galaxies are similarly close to exponential. Third, a morphology density relation exists such that dSphs are clustered around the primary galaxies while dIrrs are found at much larger distances from the them. Interestingly, Karachentsev (these proceedings) finds that the fraction of dSphs over the total number of dwarf in several nearby groups (including the Local Group) decreases drastically at distances larger than about $250 \mathrm{kpc}$ from the primary galaxies, these being comparable to the virial radius of haloes hosting bright spiral galaxies in LCDM models. This clearly suggests that only dSphs are bound satellites of the primaries and thus the environment must be playing a crucial role in differentiating dIrrs from dSphs. Of course there are outliers like the Local Group dSphs Cetus and Tucana, located at more than $500 \mathrm{kpc}$ from, respectively, M31 and the MW, but this is seen also in cosmological simulations, where a few satellites on very plunging orbits can have apocenters exceeding the virial radius of the primary (Ghigna et al. 1998).

Mayer et al. (2001a,b) and MA05 have shown that repeated tidal shocks at pericenters of their orbits within the halo of a massive spirals can transform disky dwarfs into objects resembling dSphs. The timescale of the transformation is a few orbital times (several Gyr). The mechanism behind the transformation has to do with non-axisymmetric instabilities of stellar disks. First, tidal shocks induce strong bar instabilities in otherwise stable, light disks resembling those of present-day dIrrs. Second, the bar buckles due to the amplification of vertical bending modes and turns into a spheroidal component. Finally, tidal heating/tidal mass loss thicken/remove the disk outside the buckled bar. We stress that stellar mass loss can be minimal when the disk sits deep in the potential well of a massive halo (see the GR8 run in Mayer et al. 2001b) and yet the transformation occurs. The remnants have nearly exponential profiles; the brightest among them, those coming from progenitors having relatively massive disks $\left(>10^{8} M_{\odot}\right)$, develop a central steepening of the profile due to the particularly strong bar, and overall their profile resembles that of the bright dwarf elliptical satellites of M31, like NGC205. Most importantly, the bar sheds angular momentum outwards and as a result the systems end up with low rotation, with a typical final $v / \sigma<0.5$. The transformation of course needs the dwarf to be on a bound orbit; for the mean apocenter/pericenter ratio found in cosmological simulations, $\sim 5$, Mayer et al. (2001b) determine that an apocenter distance comparable to the distance of Leo I $(250 \mathrm{kpc})$, the farthest dSph satellite of the MW, is a limiting case for producing a dSph from a disky dwarf in less than 10 Gyr (Tucana and Cetus should thus be on radial orbits).

In brief this "tidal stirring" accounts for most of the similarities and differences between dIrrs and dSphs, including the existence of the morphology density relation, by postulating progenitors of dSphs with light, low surface brightness disks embedded in massive halos like dIrrs. This does not mean that such progenitors were identical to present-day dIrrs. Since they formed at high $z$ they likely had assembly histories quite different from present-day dIrrs, and thus their stellar populations and metallicities were probably different. The missing piece now is how to place this model in the context of hierarchical structure formation. Unfortunately, to date cosmological simulations with hydrodynamics have not allowed a robust analysis of the structural evolution of satellites due to their limited resolution (typically the force resolution is $500-1 \mathrm{kpc}$, so the tiniest dSphs like Draco are not resolved at all). Searching for evidence of tidal stirring 




Figure 3. Gas mass fraction (relative to the stellar mass) of simulated dwarf galaxy satellites plotted againts the initial peak velocity of the model galaxies. Data are taken from the N-Body/SPH simulations described in MA05 plus new runs employing a local, time-dependent UV radiation field (see section 4). Measurements are done after 2 orbits within the Milky Way halo. The lower limit on the vertical axis corresponds to the resolution limit in the simulations (therefore points at the very bottom correspond to zero gas mass as measured in the simulation). Some symbols have been displaced along the horizontal axis to avoid overlaps. Filled symbols refer to runs with pericenters of $50 \mathrm{kpc}$, open symbols refer to runs with pericenters of $30 \mathrm{kpc}$. Large symbols are for galaxies with a disk gas mass fraction of 0.9 , small symbols are for those with a disk gas mass fraction of 0.3 (see section 4). Red is used for adiabatic runs, blue for runs with radiative cooling, black for runs with radiative cooling+cosmic UV background and yellow for runs with cooling, UV and star formation. The cross and star symbols are used for two runs with radiative cooling and a local UV radiation field yielding a maximum heating rate of, respectively, 5 times and 10 times the cosmic one.

in these simulations is thus quite hard, but it can be done for a few well resolved dwarfs. We selected the brightest satellites $\left(M_{B}>-14\right)$ of the large spiral galaxy in the cosmological hydro simulation of Governato, Mayer et al. (2004, hereafter GM04) as well as similarly bright dwarfs outside the virial radius of the same galaxy. Figure 2 shows their $v / \sigma$ within the effective radius versus the number of orbits performed within the main system. Clearly $v / \sigma$ correlates well with the number of orbits as expected within the tidal stirring scenario. We also found that the shape of the stellar components of the dwarfs goes from more disky to more spheroidal with increasing number of orbits. The absolute values of the $v / \sigma$ have to be taken with caution because numerical two-body heating (e.g. Mayer 2004) is certainly an issue for these objects (halos have only a few thousand particles at this scale). However the trend is evident.

\section{Gas stripping; ram pressure, tides and ultraviolet radiation}

Mayer et al. (2001b) showed that tidal stripping alone could not produce the low gas fractions found in dSphs starting from a gas-rich disky dwarf. Gas consumption by star 
formation does not change the results significantly for mean star formation rates consistent with those inferred for dSphs (Hernandez et al. 2000). MA05 study the combined effect of ram pressure and tidal stripping. They construct two-component models for the Milky Way halo in which a dark matter halo consistent with the results of LCDM simulations has an embedded diffuse gaseous component with a temperature of $\sim 10^{6} \mathrm{~K}$ and a density of about $\sim 10^{-4}$ atoms $/ \mathrm{cm}^{3}$ at $30 \mathrm{kpc}$ from the center, consistent with the values inferred from OVI absorption measurements and the existence of the Magellanic Stream (Sembach et al. 2003). Dwarf galaxies are placed on eccentric orbits with pericenters of 30 or $50 \mathrm{kpc}$. In these simulations gas-rich disky dwarfs $\left(M_{\text {gas }} / M_{\text {stars }} \geqslant 0.4\right)$ embedded in massive dark haloes typically lose $90 \%$ of their gas content. In fact ram pressure increases by a factor up to 10 the amount of stripped gas mass compared to the case in which only tides are included. Ram pressure strongly depends on the depth of the potential well of the dwarfs. While for dwarfs with $V_{\text {peak }} \leqslant 30 \mathrm{~km} / \mathrm{s}$ most of the gas content is easily removed, for more massive dwarfs the end result depends a lot on the orbit and on the temperature evolution of their gas (see Figure 3 ). The pericenter distance sets the strength of the ram pressure force mostly through the dependence on the orbital speed of the galaxy at pericenter. The temperature evolution determines whether the gas component stays extended or becomes concentrated in the central, deeper part of the potential well as a result of tidally induced bar-driven inflows. Compression from the outer medium heats the gas. The gas is rapidly heated to $10^{5} \mathrm{~K}$, where the cooling function peaks (the initial temperature is $\sim 8000 \mathrm{~K}$ ) at which point it cools radiatively to $10^{4} \mathrm{~K}$ in a fraction of the dynamical time; this cold gas easily sinks towards the center due to the torque exerted by the bar and cannot be removed by ram pressure. Instead, if compressional heating is not radiated away, as when the gas evolves adiabatically, the increased pressure opposes the bar-driven inflow, keeping more gas at larger radii where the it is more easily stripped.

For stripping to be very effective for dwarfs with initial $V_{\text {peak }} \sim 40-50 \mathrm{~km} / \mathrm{s}$ it is sufficient that the temperature of the gas is kept above $10^{4} \mathrm{~K}$ (the virial temperature of these halos is $3-5 \times 10^{4} \mathrm{~K}$ ). This requires some heating source to counteract radiative cooling. MA05 find that the (uniform) cosmic UV background at $z>2$ (Haardt \& Madau 1996) can achieve this. MA05 find that if the progenitors of Draco or Ursa Minor fell into the Milky Way at $z>2$ then ram pressure combined with tides was able to remove their entire gas content in a couple of orbits. This translates into a timescale of about 2-3 Gyr for a reasonable orbital time (namely one consistent with the current orbital distance). In this scenario the observed truncation of the star formation in Draco and Ursa Minor more than 10 Gyr ago occurred as a consequence of the infall of these galaxies into the Milky Way halo. Draco would have formed most of its stars before infall since most of the gas becomes ionized while approaching pericenter for the first time.

However, MA05 also find that the exact gas fraction of gas that becomes ionized and then stripped is quite sensitive on the initial gas density (Figure 3). Starting from initial conditions having an exponential disk comprising about $4 \%$ of the virial mass (comparable to disk mass fractions of dIrrs), a disk with $30 \%$ gas becomes entirely ionized while one with $90 \%$ remains neutral in the center and is not stripped. The mass of neutral gas could produce a stellar mass comparable to the present-day luminosity of Draco assuming star formation goes on for another few Gyr. This would yield a star formation history more extended than that of Draco, possibly closer to that of Carina and Fornax. On the other end a progenitor with a mostly gaseous disk that undergoes complete stripping is an attractive case since it would naturally produce the large $M / L$ of Draco without the need of invoking an extremely low disk mass fraction in the initial conditions (MA05). One obvious solution is to make the case for a higher heating and photoionization rate; no 
a dramatic increase of the rates would be needed since the neutral gas has a temperature only $20 \%$ lower than $10^{4} \mathrm{~K}$ in the gas rich models. The local UV flux coming from the primary galaxy during a phase of intense star formation could have been stronger than the average cosmic background. Mashchenko et al. (2004) calculate that the FUV flux of M31 derived from its $H_{\alpha}$ luminosity is higher than the present-day value of the cosmic UV background out to 10-20 kpc from its center. Current starbursts have FUV luminosities $10^{3}-10^{4}$ higher than the MW and M31, in the range $10^{44}-10^{45} \mathrm{erg} / \mathrm{s}$ (Leitherer et al. 2002). According to cosmological simulations at $z=2-3$ the Milky Way was hosting a major central starburst as the bulge was being assembled from a big merging event (GM04). A starburst is also predicted by models of the star formation history in the galactic bulge, which yield peak star formation rates of up to $100 M_{\odot} /$ yr (Elmegreen 1999), comparable to the most spectacular of present-day starbursts. Lower quantities of dust expected given the lower metallicity of the gas at high redshift imply an escape fraction of ultraviolet photons higher than that in present-day starbursts. But let us be conservative and just assume that the FUV luminosity of the bulge was comparable to that of a major present-day starburst $\left(L_{F U V}=10^{45} \mathrm{erg} / \mathrm{s}\right)$. Under this hypothesis one obtains that at $30 \mathrm{kpc}$ from the bulge the heating rate was 10 times higher than that associated with the metagalactic UV background at $z=2-3$. We have run new simulations including such a local, time-dependent UV background (the intensity of the flux is modulated by the orbital distance of the dwarf) and found that ram pressure stripping at the first pericenter passage can be greatly enhanced in a gas-rich dwarf. Even the most dark matter dominated among our models, with $V_{\text {peak }}=40 \mathrm{~km} / \mathrm{s}$, loses more than $90 \%$ of its gas along the first orbit and the remaining gas is completely ionized. The ionized gas is then stripped along the second orbit (Figure 3). This simulation reproduces naturally both the early truncation of the star formation in Draco and its present-day high $M / L$ ratio $(>100)$.

\section{Towards a coherent picture; from gas stripping and gas inflows to the star formation histories}

A coherent picture is emerging. Dwarf spheroidals live in tidally truncated halos with moderate masses, $10^{8}-10^{9} M_{\odot}$, yet this implies $M / L>100$ in some of them (e.g. Draco and Ursa Minor). However, since simulations show that most of the dark matter halo was stripped as the dwarfs entered the primary halos, their original dark matter content was much higher. Tidal stripping can remove mass even quite close to the center, reducing their $V_{\text {peak }}$ by 30-50\% (see Figure 1). The tidal interaction with the massive halos of the primaries is also responsible for the morphological transformation of small disks into spheroidal systems. Gas stripping instead is the result of the combination of tidal and ram pressure stripping. However a heating source is required in order to keep the gas extended and allow efficient stripping for galaxies with $V_{\text {peak }}>30 \mathrm{~km} / \mathrm{s}$. The more attractive scenario is one in which the heating was provided by the cosmic UV background at high redshift aided by an even stronger local FUV field produced by the primary galaxies during an epoch of intense star formation. According to galaxy formation models this epoch should coincide with the time of bulge formation, at $z \geqslant 2$. This would explain the sudden truncation of star formation that occurred in Draco and Ursa Minor about 10 Gyr ago. Dwarfs that entered the Milky Way halo later would have kept some gas for at least one or two orbits and thus undergo extended star formation. Our simulations show that the remaining gas falls towards the center as a result of bar driven inflows at pericenter passages. The central gas density there increases and a burst of star formation will occur, as shown in Mayer et al. (2001b). This explains the bursty star formation histories of 
Carina or Fornax. Ram pressure and tidal stripping are reduced significantly at more recent epochs for two reasons; the first is that in $\Lambda$ CDM models orbits of satellites have on average larger pericenters and longer orbital times at more recent epochs (see the cosmological simulations of Ghigna et al. (1998) for example), the second is that both the local and cosmic UV background fade by orders of magnitude at $z<2$. In summary we predict that dSphs with extended or truncated star formation histories come from similar progenitors that fell into the primary halos at different epochs. One variable whose evolution with time is not well known is the density of the hot gaseous corona of the Milky Way which sets in part the strength of ram pressure. However new simulations of galaxy formation currently in progress suggest that the density of such halo was higher at higher redshift, which would reinforce our scenario.

\section{Acknowledgements}

I thank Stelios Kazantzidis, Chiara Mastropietro, Ben Moore and Fabio Governato for the many stimulating discussions on dwarf galaxies and Cristiano Porciani for his help on issues regarding the effects of the ionizing radiation.

\section{References}

Bullock, J.S., Kravtsov, A.V. \& Weinberg, D.H. 2000, ApJ 539, 517

Dekel, A. \& Silk, J. 1986, ApJ 303, 39

Einasto, J., Saar, E., Kaasik, A. \& Chemin, A.D. 1974, Nature 252, 111

Elmegreen, B. 1999, ApJ 517, 103

Ghigna, S., Moore, B., Governato, F., Lake, G., Quinn, T. \& Stadel, J. 1998, MNRAS 300, 146

Governato, F., Mayer, L., Wadsley, J., Gardner, J.P., Willman, B., Hayashi, E., Quinn, T., Stadel, J. \& Lake, G. 2004, ApJ 607, 688

Grebel, E.K. \& Gallagher, J.S. 2004, ApJ 610, L89

Haardt, F. \& Madau, P., 1996, ApJ 461, 20

Hernandez, X., Gilmore, G. \& Valls-Gabaud, D. 2000, MNRAS 317, 831

Kazantzidis, S., Mayer, L., Mastropietro, C., Diemand, J., Stadel, J. \& Moore, B. 2004, ApJ 608,663

Kravtsov, A.V., Gnedin, O.Y. \& Klypin, A.A. 2004, ApJ 609, 482

Leitherer, C., I-Hui, L., Calzetti, D. \& Heckman, T.M. 2002, ApJ 303

Mac Low, M. \& Ferrara, A. 1999, ApJ 513, 142

Mashchenko, S., Carignan, C. \& Bouchard A. 2004, MNRAS 352, 168

Mateo, M. 1998, ARA $\& A$ A 36, 435

Mayer, L., Governato, F., Colpi, M., Moore, B., Quinn, T., Wadsley, J., Stadel, J. \& Lake G. 2001a, ApJ 547, L123

Mayer, L., Governato, F., Colpi, M., Moore, B., Quinn, T., Wadsley, J., Stadel, J. \& Lake G. 2001b, ApJ 559, 754

Mayer, L., Moore, B., Quinn, T., Governato, F. \& Stadel, J. 2002, MNRAS 336, 119

Mayer, L., Mastropietro, C., Wadsley, J., Moore, B., \& Stadel, J. 2005, MNRAS submitted, astro-ph/0504277

Moore, B., Ghigna, S., Governato, F., Lake, G., Quinn, T., Stadel, J. \& Tozzi, P. 1999, ApJ 524, L19

Sembach, K.R. et al. 2003, ApJS146, 165

Stoehr, F., White, S.D.M., Tormen, G. \& Springel, V. 2002, MNRAS 335, L84

Susa, H. \& Umemura, M. 2004 ApJ 600, 1

Wilkinson, M., Kleyna, J.T., Evans, W.N., Gilmore, G.F., Irwin, M.J. \& Grebel, E.K. 2004, ApJ 611, L21 


\section{Discussion}

Dekel: How do you explain Tucana and Cetus? 2) How do you explain the continuous $\mathrm{SFH}$ in some dwarfs?

MAYeR: We don't know the orbits of Tucana and Cetus. They could be nearly radial, and one perigalactic passage is enough to strip most of the gas with tides and ram pressure. You see a few subhalos on such orbits in CDM simulations. Also, Kravtsov et al. (2004) have shown that you can get tidal stirring also before dwarfs enter the MW halo in a few cases. 2) Tidal stirring does predict periodic, extended SFHs. You need to add ram pressure stripping for those satellites that do not have extended SF, like Draco and UMi. So, if you have tides and ram pressure you can have a truncated or an extended SFH depending on (i) the initial mass of the satellite and (ii) its orbit. therefore I think you can get the different SF histories of dSphs quite naturally.

Skillman: Eline Tolstoy has measured a large radial velocity for Tucana implying it would have been close to the Local Group barycenter about 5 Gyr ago (about when star formation ceased), in which case stripping is a possibility.

MAYER: This would be consistent with CDM simulations as well. You always see a few satellites on very plunging orbits as Tucana. If Tucana has a DM halo of $<10^{9} M_{\text {sun }}$ then one passage would be enough for tides and ram pressure to strip most of the gas.

Kroupa: Can your model also account for the lack of MW-to-LMC-sized satellites in fossil groups, as found by D'Onghia \& Lake (2004)? 2) Can your model remove the DM cusps in MW satellites? Kazantzidis et al. (2004) find this is not possible.

MAYER: I think the luminosity function of the fossil groups is not known well enough. Only a few objects have been studied and I do not think we know the substructure mass function down to the LMC scale. 2) No, cusps always stay.

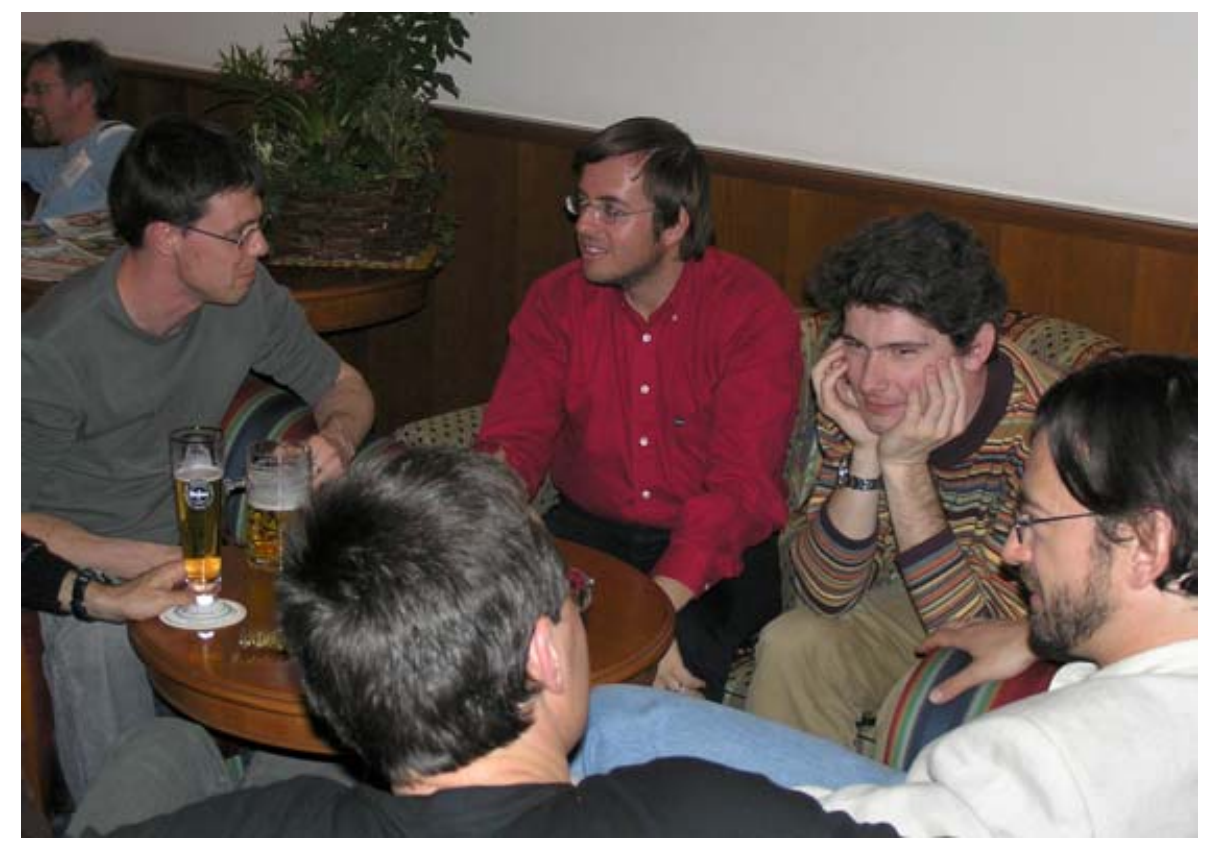

